\title{
Deciphering complex, functional structures with synchrotron-based absorption and phase contrast tomographic microscopy
}

\author{
M. Stampanoni*a,b ${ }^{\text {a. }}$. Reichold ${ }^{\mathrm{c}}$, B. Weber ${ }^{\mathrm{d}}$, \\ D. Haberthür ${ }^{\mathrm{e}}$, J. Schittny $^{\mathrm{e}}$, J. Eller ${ }^{\mathrm{f}}$, F. N. Büchi ${ }^{\mathrm{f}}$, F. Marone ${ }^{\mathrm{a}}$

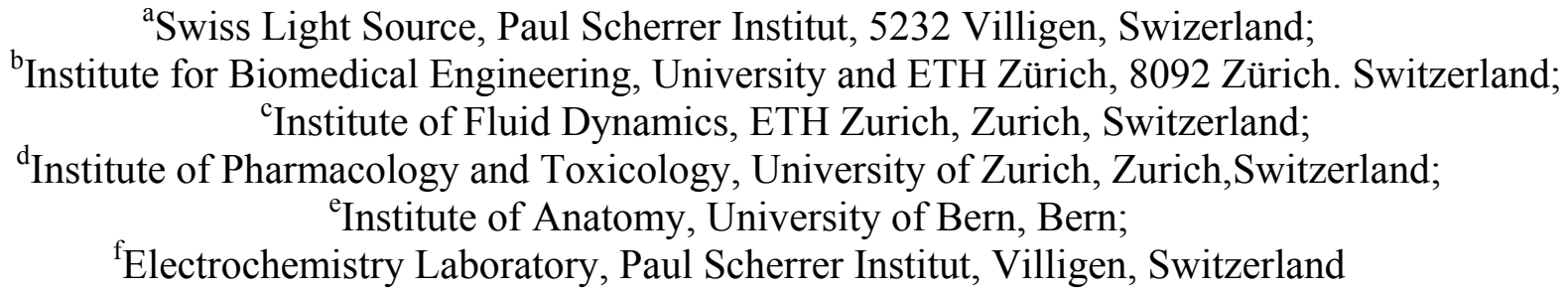

\begin{abstract}
Nowadays, thanks to the high brilliance available at modern, third generation synchrotron facilities and recent developments in detector technology, it is possible to record volumetric information at the micrometer scale within few minutes. High signal-to-noise ratio, quantitative information on very complex structures like the brain micro vessel architecture, lung airways or fuel cells can be obtained thanks to the combination of dedicated sample preparation protocols, in-situ acquisition schemes and cutting-edge imaging analysis instruments. In this work we report on recent experiments carried out at the TOMCAT beamline of the Swiss Light Source [1] where synchrotron-based tomographic microscopy has been successfully used to obtain fundamental information on preliminary models for cerebral fluid flow [2], to provide an accurate mesh for 3D finite-element simulation of the alveolar structure of the pulmonary acinus [3] and to investigate the complex functional mechanism of fuel cells [4]. Further, we introduce preliminary results on the combination of absorption and phase contrast microscopy for the visualization of high- $Z$ nanoparticles in soft tissues, a fundamental information when designing modern drug delivery systems [5]. As an outlook we briefly discuss the new possibilities offered by high sensitivity, high resolution grating interferomtery as well as Zernike Phase contrast nanotomography [6].
\end{abstract}

Keywords: X-ray tomographic microscopy, cerebral blood flow, lung imaging, phase contrast, nano-imaging

\section{INTRODUCTION}

This article describes, based on few examples, how state-of-the art synchrotron-based tomographic microscopy data constitute nowadays a fundamental tool for tackling complex challenges in biomedical, energy and materials sciences. During the last few decades, with the development of synchrotron sources, X-ray imaging has experienced a true revolution. Within a few years, the brilliance of X-ray sources has been increased by more than 10 orders of magnitude, making coherent, very intense X-ray beams available. The tremendous photon density reached by these novel sources has pushed X-ray imaging to the level of X-ray microscopy and further to tomographic microscopy. Further, and probably the most important characteristic of such coherent beams, is their intrinsic capability of generating interference

*marco.stampanoni@psi.ch; phone+41 5631047 24; www.psi.ch

Developments in X-Ray Tomography VII, edited by Stuart R. Stock, Proc. of SPIE Vol. 7804, 78040L · @ 2010 SPIE · CCC code: 0277-786X/10/\$18 · doi: 10.1117/12.860208 
signals and, as a consequence, to provide access to phase information in the investigated sample. This property is particularly useful when imaging poorly absorbing specimens. The Swiss Light Source (SLS) at the Paul Scherrer Institute (PSI) in Switzerland operates TOMCAT, a beamline dedicated to TOmographic Microscopy and Coherent rAdiology experiments [1]. This beamline provides cutting-edge equipment for tomographic experiment and in particular offers the necessary instrumentation for phase contrast imaging at spatial resolution ranging over three orders of magnitude [7].

The paper focuses first on how high-resolution tomographic microscopy data can be used to generate a vascular graph model for the simulation of blood flow. In a second example we describe how finite-element (FE) techniques can be applied to reconstruct the pulmonary acinus. On this subject, we report on a recently developed method to expand the lateral field of view of tomographic microscopy which can be particularly suited for imaging functional units like a lung acinus. In a further section, we show how phase contrast tomographic microscopy has been successfully applied to reveal complex structure within gas diffusion layers, a fundamental component of a fuel cell. In the last section we briefly update about the phase contrast capabilities of TOMCAT.

\section{SIMULATION OF CEREBRAL BLOOD FLOW}

\subsection{Background and Motivation}

Cerebral blood flow (CBF) is dynamically regulated to ensure that supply and current metabolic demand are always in balance. Recently, imaging modalities have been developed that use CBF as a surrogate of neural activity for functional mapping [8], [9]. However, several studies have shown that neurovascular coupling is a nonlinear phenomenon, and the spatial specificity of the hemodynamic response, as well as the complex interplay of the diverse CBF regulating mechanisms remain largely unknown [10]. The current state of the art blood flow imaging modalities are either limited to a small field of view or have a low spatial and/or temporal resolution. Computational modeling of CBF does not suffer from these limitations, and has become an important research tool which provides an alternative method to test hypotheses in ways that are not possible in experimental investigations. General models introduced so far, either make highly simplified approximations of the angioarchitecture or focus on a small fraction of the cerebral vasculature only, for example, the microvascular network [11] or large vascular structures, such as the Circle of Willis [12]. Boas et al. presented a very simple model that can accurately predict the dynamic behavior of the complex vascular system [13] with no restrictions either to a specific imaging method or to a particular fraction of the vasculature. Recently Reichold et al., introduced a vascular graph (VG) model based on the same philosophy but applied to realistic vascular networks as, for instance, obtained by high-resolution angiography [2]. The model introduced by Reichold et al., and subject of this section, suggests several upscaling algorithms that allow working with integral quantities of the capillary bed, rather than with its discrete representation: this is very useful when the discrete capillary topology is unknown and obviously significantly reduces the computational costs.

\subsection{Methods}

\section{Sample Preparation and Tomographic Microscopy}

Deeply anesthetized rats were transcardially perfused with heparinized phosphate buffered saline followed by $4 \%$ paraformaldehyde. Then, a dispersed suspension of $\mathrm{BaSO}_{4}$ was injected. After removal of the brain, cylindrical samples of approximately $2.8 \mathrm{~mm}^{3}$ from the somatosensory cortex were punched out and embedded in EPON [14]. Tomographic microscopy has been done with beam energy of $20 \mathrm{keV}$ to maximize absorption contrast and to provide sufficient photon flux to penetrate the large sample. The optical magnification was $20 \mathrm{x}$, resulting in isotropic voxels of $370 \mathrm{~nm}$ for the reconstructed images.

\section{Base Model Concepts}

The basic idea is to represent the cerebral vasculature by a graph, i.e., a collection of vertices or 'nodes' and an ensemble of edges that connect pairs of nodes. In such a vascular graph (VG) model, nodes represent locations in which vessels bifurcate or end, and edges designate the vessels themselves. In graph-theory terminology, the cerebral vasculature can be called a multigraph because two vertices may be connected by more than one edge. The graph data structure provides an easy handle to compute CBF. If two adjacent nodes (nodes that share a common edge) are at different blood pressure values, this will elicit a blood flow in the vessels that connect them. The magnitude of the flow will depend on the 
pressure difference and the conductance of the vessels. If mass conservation at each node is imposed, the system of equations to yield pressure and flow values for the entire graph (given appropriate boundary conditions) can be solved.

\section{Image post-processing, vessel classification and model generation}

After segmentation, blood vessels midlines are extracted and branching and end points of the thinned vessels are determined. Each midline is associated with a vessel radius value. The tortuous midlines are further replaced by straight edges. Each edge is assigned a radius value, which is the mean of the radii associated with the corresponding midline voxels. As a consequence, the graphical representation of an edge is a cylinder with a radius equal to the assigned mean radius, see Figure 1. Large vessels were classified as either arteries or veins, based on the results of a pair-wise connectivity analysis. Vessels that connect strongly to each other were assigned the same category, based on the fact that no arterio-venous shunts exist in healthy brain. Arteries or veins have been identified on topological criteria, like vessel diameter or number (veins are larger than arteries) of vessel branching (veins have more daughter vessel at similar blunt angles).
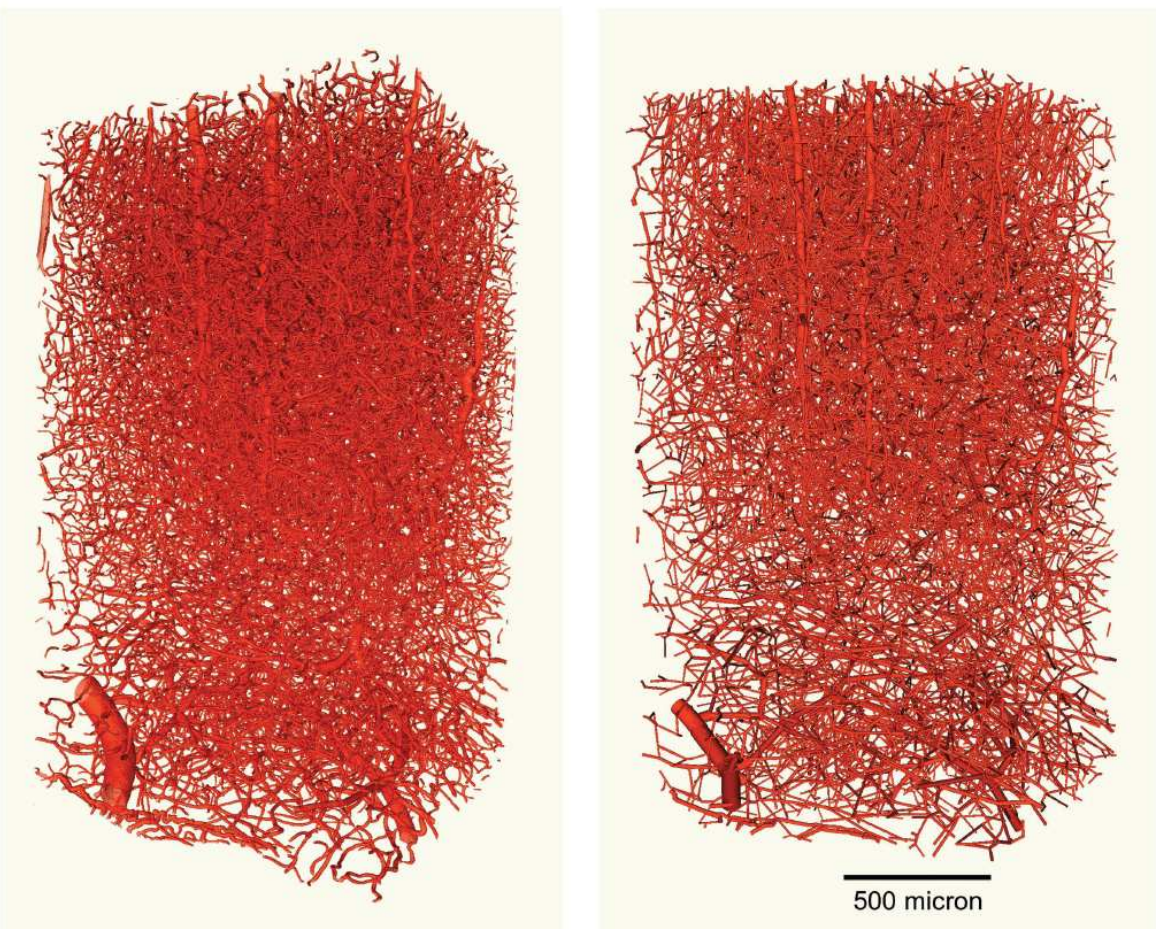

Figure 1: Left: Synchrotron-based tomographic microscopy data of the rat somatosensory cortex. Right: Vascular graph model representation of the same data set. The tortuous vessel parts have been replaced by straight connections. Note that the radii of all edges were scaled such that a mean capillary size of 6 microns was reached yielding a vascular volume fraction of $2.52 \%$. This step is necessary to account for shrinkage of the perfused brain tissue during the preparation process. (C) Journal of Cerebral Blood Flow \& Metabolism.

The derivation of the equations governing the model has been carried out by Reichold et al. [2] and goes beyond the scope of this paper. At least however, it has to be mentioned that to support the validity of the cylinder-approximation model, one can look at measurements of the blood flow velocities in cortical vessels [15], which show that Reynolds number $R e \leq 2$ for all but the largest vessels in the brain. This implies that inertial effects play an insignificant role and that the cross-sectional velocity profile remains stable even for curved paths. Every node is further associated with a blood pressure value and differences in pressures between adjacent nodes drive the flow through the vessels connecting them. The conductance of such a vessel is modeled as $G=\frac{\rho A^{2}}{8 \pi \mu L}$ where A, L, $\mu$ and $\rho$ are the cross-sectional area and length of the vessel, the dynamic viscosity and blood density respectively. Consequently, the mass flow rate $\mathrm{F}$ through a vessel can be expressed as the product of its conductance and the pressure difference across its length: $F=G \Delta p$. 
The continuity equations states that the net mass flow through every node $i$ must match the local accumulation rate (unless the node is a sink or a source) and therefore, for each node, one can write:

$$
\frac{\partial V_{i}}{\partial t}+\frac{1}{\rho} \sum_{j} G_{i j}\left(p_{i}-p_{j}\right)=\frac{q_{i}}{\rho}
$$

This sparse linear equation system can be solved provided appropriate boundary conditions are given, see details in [2].

\subsection{Results}

Reichold et al. [2] investigated different scenarios and studied the effects of local vascular dilation and occlusion on the flow in the surrounding network. Figure 2 shows an example where an occlusion scenario was created by constricting the diameter of a feeding artery to $1 / 1000$ of its original size. The simulation indicates that the occlusion of an arteriole has a marked effect on the blood flow in the capillary bed it irrigates, as well as on the veins to which it is most strongly linked to. Blood flow slowly recovers towards baseline with increasing numbers of bifurcations away from the occluded penetrating arteriole as the influence of adjacent feeding arterioles increases. However, at 6 to 10 bifurcations away from the occluded vessel, flow values are still lower than half baseline. Moreover, a flow increase in the surrounding arterioles of around $3.6 \%$ can be observed. This change, however, cannot compensate the severe drop in CBF in those regions that were irrigated by the occluded arteriole.
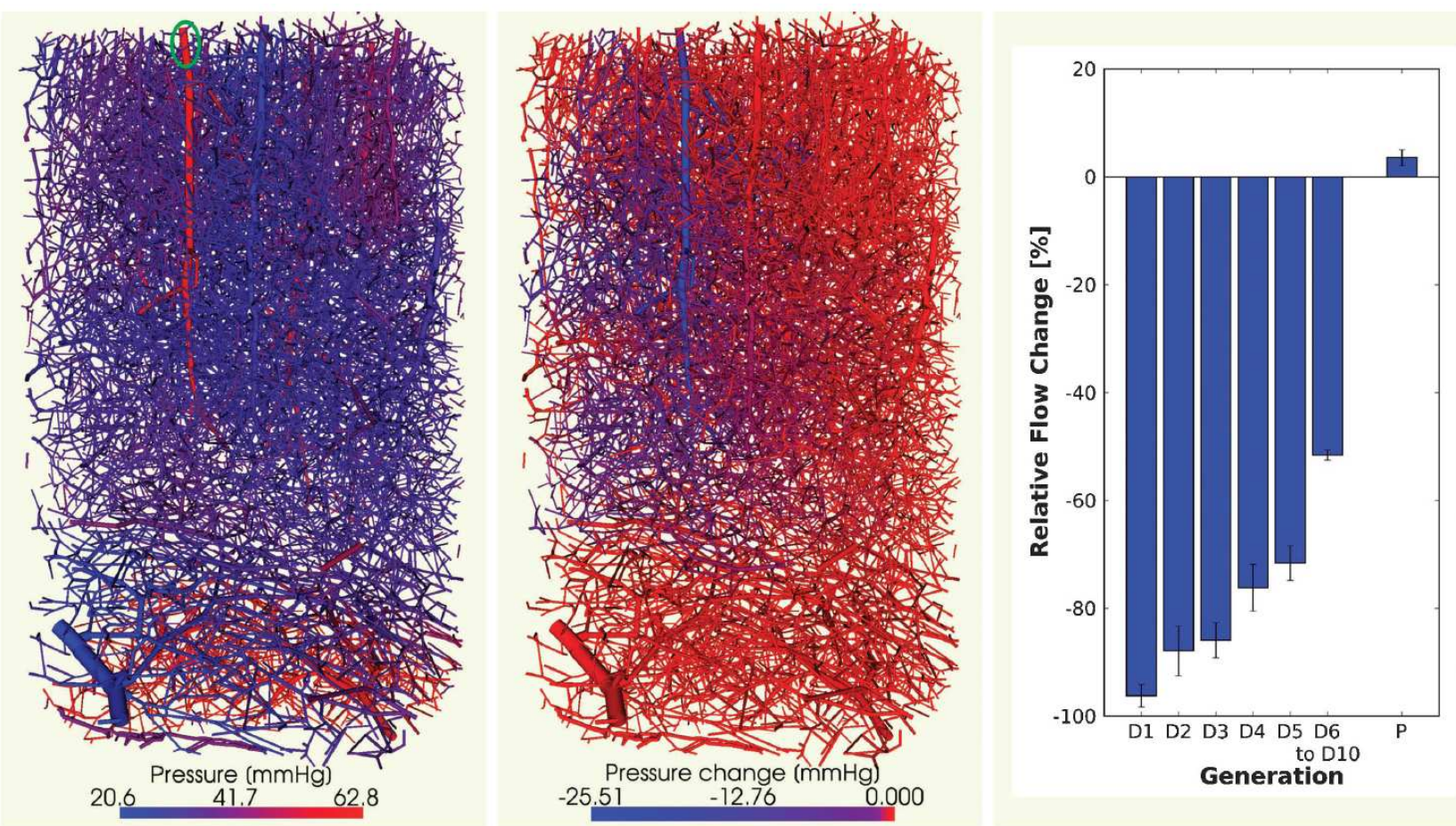

Figure 2: Occlusion of a penetrating cortical arteriole. Left: initial vascular network color-coded by pressure. A green ellipse indicates the site of the occlusion. Middle: change in pressure due to the occlusion. Right: blood flow change categorized by topology. D1 denotes the first generation of vessels that branch off the penetrating arteriole, D2 denotes vessels that branch off the D1 generation and so on. Penetrating arterioles parallel to the occluded arteriole fall under category P. The error bars indicate the standard error of the mean (C) Journal of Cerebral Blood Flow \& Metabolism.

The framework suggested by Reichold et al. [2] models the flow of blood through the cerebral vasculature, based on simple fluid dynamics principles. It includes fully three-dimensional advective transport, vasculature-tissue exchange and diffusion within the tissue, allowing to simulate oxygen transport, drug delivery and alike. Upscaling algorithms facilitate its application to large-scale networks and dynamic systems. The VG framework provides a means to 
investigate the flow properties of case (patient) specific vascular networks. Moreover, it can be used as a powerful tool to research the effects of vascular changes on the cerebral blood flow.

\section{VISUALIZATION AND MODELING OF THE PULMONARY ACINUS}

\subsection{Background and motivation}

The mammalian inter-thoracic respiratory tract can be divided into two areas: conducting airways and pulmonary parenchyma (i.e., the gas exchange region of the lung). The conducting airways are a bifurcating network of relatively well-defined conduits carrying the ambient air to the parenchyma. Airways within the parenchyma -- customarily called acinar/alveolar ducts -- on the other hand, are not pipe-like and they are formed by entrance rings of alveoli opening into a common passageway. In contrast to conducting airways, lung parenchyma is hard to access since the structures are physically small and located distally in the respiratory tract. Basic structures of lung parenchyma are highly complex as can be expected by the fact that the enormously large surface area of lung parenchyma -- as large as that of a tennis court in the case of human lungs [16] -- has to be folded in within the lung cavity. Synchrotron-based tomographic microscopy has been proven to be a suitable tool for the visualization of lung structures, down to the thin alveolar septa [17]. The motivations for reconstructing the 3D geometry of the respiratory region of the lung are numerous. Two-dimensional (2D) sections of lung parenchyma are insufficient for many purposes and can produce erroneous models of lung structure [18]. 3D structural rendering is crucial to the study of micromechanics in 3D acinar microarchitecture, particularly given the likelihood of heterogeneous behavior with ventilation. Only by using a realistic 3D structure (for example, of alveolar shape and orientation of the alveolar opening with respect to the central thoroughfare acinar channel) the qualitative and quantitative effects of 3D acinar geometry on gas and aerosol transport can be determined.

\subsection{Methods}

\section{Visualization of large volumes at high resolution}

In parallel-beam-geometry tomographic experiments, X-ray projections are usually taken at equidistant angles over a sample rotation of $180^{\circ}$. After reconstruction, the width of the image corresponds to the field of view of the camera. Samples that are twice as large as the field of view, can be imaged using scanning protocols based on a $360^{\circ}$ off-center sample rotation. Images recorded between $180^{\circ}$ and $360^{\circ}$ are flipped and the projections obtained at angular position $\theta$ and $\theta+180^{\circ}$ are stitched into one projection. The resulting images cover twice the field of view of the camera. For tomographic scans covering a size wider than two fields of view, D. Haberthür et al. [19] recently suggested a method dubbed "wide-field scanning" that allows a (radiation dose optimized) lateral expansion of the field of view. In the simplest case, three $180^{\circ}$-scans are taken at slightly overlapping positions and combined. The cutline, i.e. the position where the merging takes place, is automatically determined according to a mean-squared difference method [20]. Note that a "brute force" acquisition approach would record an equal amount of projections for each of the individual subscans. As a consequence, to fulfill the sampling theorem in the sample's extremities, oversampling of the central parts becomes unavoidable. Based on the assumption that sufficient resolution and contrast can be achieved if the sampling theorem is simply satisfied for each of the subscan individually, Haberthür et al. devised an acquisition scheme that can save up to $85 \%$ of the exposure time compared to the "brute force" approach. Figure 3 shows a rat lung sample imaged with the wide-field protocol. Thanks to this novel approach, large volumes can be measured with high resolution and a complex structure like the lung acinus can visualized entirely with details down to the single alveolar walls, see Figure $3 \mathrm{~h}$.

Animal preparation and tomographic microscopy.

Lungs of 129/SV mice were prepared according to Schittny et al. [21] at postnatal day 10. Briefly, the airspace was filled with $2.5 \%$ glutaraldehyde in $0.03 \mathrm{M}$ potassium-phosphate buffer (pH 7.4, $370 \mathrm{mOsm}$ ) at a constant pressure of $20 \mathrm{~cm}$ water column. At this pressure, the lung reaches roughly its mid-respiratory volume. In order to prevent a recoiling of the lung, the pressure was maintained during fixation $(\geq 2 \mathrm{~h})$. Samples were postfixed in $0.1 \mathrm{M}$ sodium cacodylate $(\mathrm{pH} 7.4$, $340 \mathrm{mOsm}$ ), containing $1 \% \mathrm{OsO}_{4}$, and stained en bloc with $0.5 \%$ uranyl acetate in $0.05 \mathrm{M}$ maleate buffer. After 
dehydration in a graded series of ethanol, the samples were embedded in Epon 812 [22]. Synchrotron-based tomographic microscopy has been performed at $1 \AA$, with an isotropic voxel size of 1.4 microns.
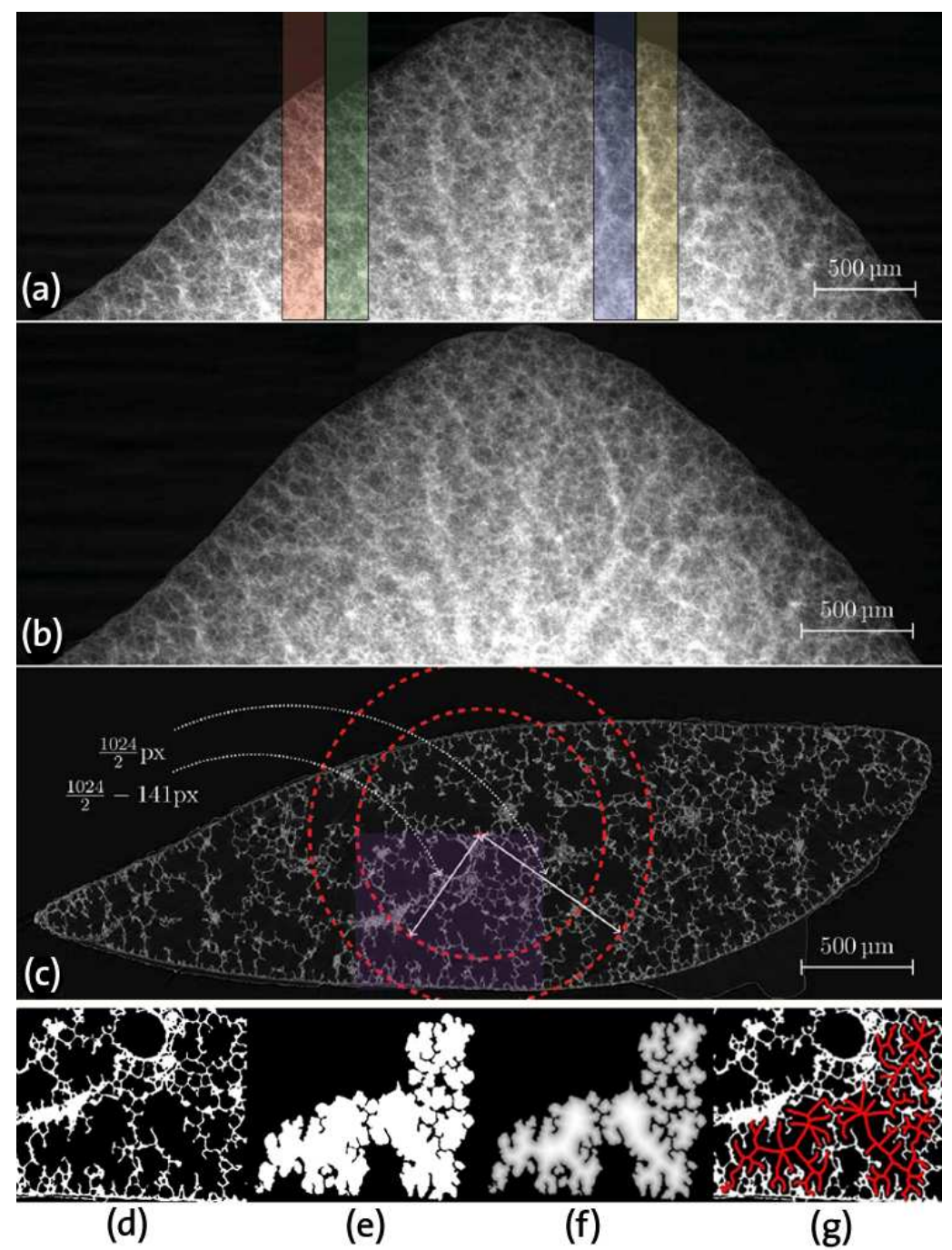

(f)

(g)

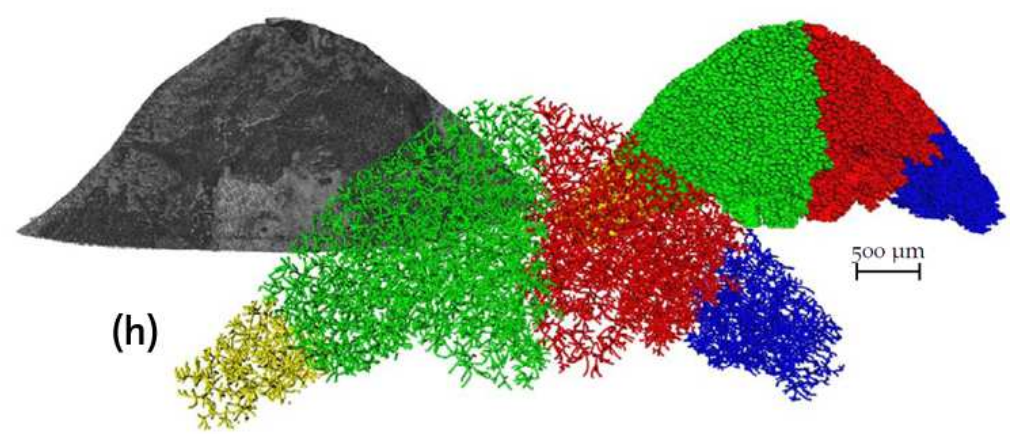

Figure 3: (a-c): The images show a rat lung sample from a Sprague-Dawley rat, obtained 21 days after birth, scanned with the wide-field approach. (a) Three baseline corrected projections from subscans s1-s3. Each one is $1024 \times 1024$ pixels large and covers a field of view of $1.52 \mathrm{~mm}$. Subscans s1 and s2 overlap by 141 pixels (red and green overlay), subscans s2 and s3 overlap by 138 pixels (blue and yellow overlay). (b) Merged projection obtained from the three subscans shown in subfigure (a). Each merged projection has a size of $2792 \times 1024$ pixels. 
(c) Reconstructed tomographic slice: the dashed red circles mark the start and end of the overlap region. (d-g): (d) Binarized (separation of tissue from air) region of interest of the area marked in violet in (c). (e) The white segment represents one connected airway structure inside the ROI in two dimensions (f): Euclidean distance transformation of the segment from panel (d). The gray level value of each pixel represents the distance to the border between tissue and airspace. (g): The local maxima of the distance transformation form the skeleton can be overlaid on the binarized lung structure (d). (h) Left: Three-dimensional view of the lung sample, Right: Four independent airway segments containing multiple acini. Foreground: Extracted airway skeletons of the independent airways. The yellow skeleton contains 1133, the green 7288, the red 6513 and the blue skeleton 3278 nodes. Images adapted from [23].

\section{Towards computational fluid-dynamics models of the breathing mechanism}

Airflow into and out of the lung is driven by pressure differences between alveoli and the outside environment. Pressure gradients are induced through the lung wall expansion and contraction motion during rhythmical breathing. The principle model of lung expansion approximately satisfies geometric self-similarity [24]. Therefore, any geometrical length scales approximately with the $1 / 3$ power of the lung volume. Sznitman et al. [25] presented a model where alveolar airspaces were designed to expand and contract in a simple sinusoidal manner, with a breathing period T. Any length scale, $\mathrm{L}(\mathrm{t})$, was then described by the kinematic displacement function:

$$
L(t)=L_{0}\left[1+\frac{\beta}{2}+\frac{\beta}{2} \cdot \sin \left(2 \pi \cdot f \cdot t-\frac{\pi}{2}\right)\right]
$$

where $L_{0}$ is the length scale at $t=0, f=1 / T$ is the breathing frequency. The specific volume excursion is given by $\mathrm{C}=\left(\mathrm{V}_{\max }-\mathrm{V}_{\min }\right) / \mathrm{V}_{\min }$, where $\mathrm{V}_{\min }$ and $\mathrm{V}_{\max }$ are respectively the minimum and maximum volumes of the alveolar sac geometries. For geometrically self-similar wall motion, the length scale expansion factor, $\beta$, is given by $\beta=(C+1)^{1 / 3}-1$ and the length scale reaches a maximum of $\mathrm{L}_{\max }=\mathrm{L}_{0}(1+\beta)$ at $\mathrm{t}=\mathrm{T} / 2$. Note that real tidal breathing does not exhibit a perfectly sinusoidal change of volume with time. Rather, the lung illustrates a small but significant geometric hysteresis [26]. Nevertheless, to the extent with which acinar flows are quasi-steady, the sinusoidal breathing approximation can be adopted [27].

In terminal acinar airspaces, flow is incompressible; therefore, the equations of fluid motion (conservation of mass and momentum) are governed by the unsteady, incompressible Navier-Stokes equations:

$$
\begin{aligned}
\nabla \cdot \underline{u} & =0, \\
\rho\left(\frac{\partial \underline{u}}{\partial t}+\underline{u} \cdot \nabla \underline{u}\right) & =-\nabla p+\mu \nabla^{2} \underline{u}
\end{aligned}
$$

where $\underline{u}$ is the fluid velocity vector field, $p$ is the fluid pressure, $\rho$ its density and $\mu$ its dynamic viscosity (being $v=\mu / \rho$ the fluid's kinematic viscosity). Sznitman et al. [25] assumed laminar flow conditions and neglected external body forces (e.g. gravity). Equations (1.3) and (1.4) are solved numerically on a moving grid using a commercial finite-volume based program with fully implicit marching techniques under isothermal conditions. Airflow is entirely generated from the wall motion induced during rhythmical breathing, by implementing the length scale displacement function described in Equation (1.2) and the appropriate boundary conditions.

Two alveolar sac geometries of variable size and complexity were investigated. The first alveolar sac geometry I (Figure 4a) consisted of approximately $280^{\prime} 000$ volume elements, corresponding to an initial volume at $t=0$ of $\sim 0.8 \cdot 10^{-3}$ $\mu \mathrm{L}$, while the larger alveolar sac II (Figure 4b) consisted of approximately 600 '000 volume elements for an initial volume of $\sim 2.7 \cdot 10^{-3} \mu \mathrm{L}$. Volume elements consisting of layers of prisms were employed to improve the resolution of the flow in the vicinity of the wall.

3D streamlines are illustrated at peak inspiration $(\mathrm{t}=0.1 \mathrm{~s})$ in Figure $4 \mathrm{c}$ and Figure $4 \mathrm{~d}$. Flow topologies in both alveolar sacs are similar, despite variations in geometrical size and complexity. Namely, flow in the terminal acinar generation is 
governed by Stokes' flow (at the duct inlet, $R e \approx 0.0001$ and $R e \approx 0.0035$ respectively for geometry I and II). Streamlines are radial, filling individual alveoli as observed in previous models of terminal airspaces [27]. Rhythmic wall expansion generates flow at the duct inlet which reaches the alveolar walls, as fluid is dragged inside the airspace due to a negative pressure gradient. Following conservation of mass, the velocity along streamlines sharply decreases as fluid travels deeper into the alveolar sac. Flow phenomenon is driven by the basic rhythmical wall motion and much less by specific features in the alveolar geometry. Flow patterns and small particle trajectories are presented in detail in Sznitman et al. $[25]$.
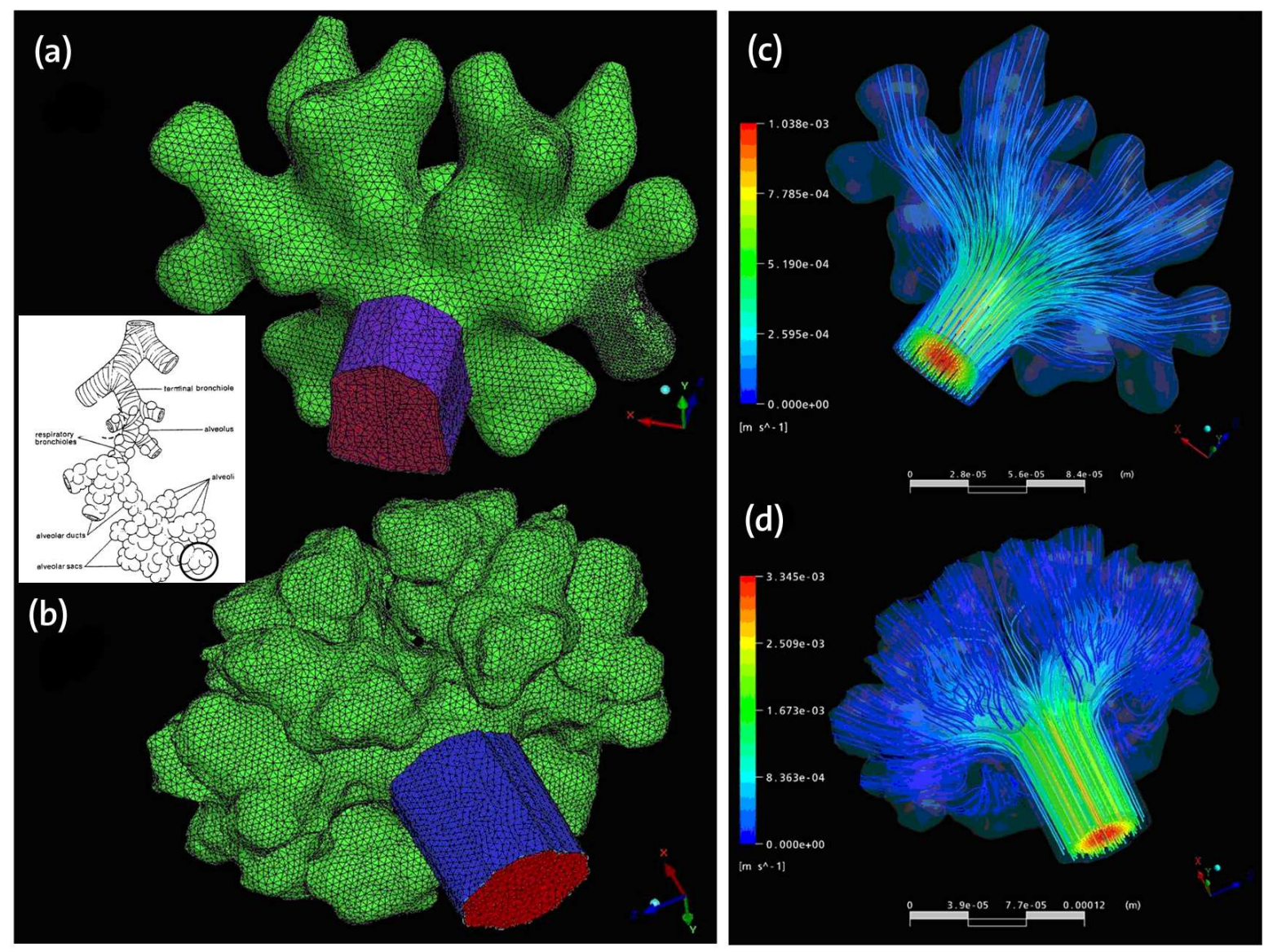

Figure 4: (a-b): Resulting meshes for the reconstructed 3D alveolar sacs are illustrated for (a) geometry I and (b) II. Both geometries are characterized by the existence of symmetry planes and the presence of a short entrance duct feeding the alveolar space (shown in a distinct color). Geometry I is composed of approximately 280 '000 volume elements and geometry II, approximately 600'000 elements. Inset: Schematic representation of the pulmonary acinus with the location of an alveolar sac circled (modified from [28]). (c-d): Illustration of instantaneous alveolar streamlines with velocity field magnitude in three-dimensional space, obtained at peak inspiration $(\mathrm{t}=0.1 \mathrm{~s})$ in alveolar sac geometries (c) I and (d) II. Color bar on left denotes velocity magnitude in $[\mathrm{m} / \mathrm{s}]$. Images modified with permission from J. Sznitman et al. [29].

\section{VISUALIZATION OF HIGH-Z NANOPARTICLES IN SOFT TISSUES}

\subsection{Background and motivation}

The role of nanotechnology in medicine and more specifically in drug delivery is spreading rapidly. Several substances are under investigation for drug delivery and more specifically for cancer therapy. Interestingly pharmaceutical sciences are using nanoparticles to reduce toxicity and side effects of drugs and up to recently did not realize that carrier systems 
themselves may impose risks to the patient, [5]. The kind of hazards introduced by using nanoparticles for drug delivery might be beyond that posed by conventional chemicals in classical delivery matrices. For nanoparticles the knowledge on particle toxicity as obtained in inhalation toxicity shows the way how to investigate the potential hazards of nanoparticles. The toxicology of particulate matter differs from toxicology of substances as the composing chemical(s) may or may not be soluble in biological matrices, thus influencing greatly the potential exposure of various internal organs. This may vary from a rather high local exposure in the lungs and a low or neglectable exposure for other organ systems after inhalation [30]. However, absorbed species may also influence the potential toxicity of the inhaled particles. For nanoparticles the situation is different as their size opens the potential for crossing the various biological barriers within the body [31]. From a positive viewpoint, especially the potential to cross the blood brain barrier may open new ways for drug delivery into the brain. In addition, the nanosize also allows for access into the cell and various cellular compartments including the nucleus. A multitude of substances are currently under investigation for the preparation of nanoparticles for drug delivery, varying from biological substances like albumin, gelatine and phospholipids for liposomes, and more substances of a chemical nature like various polymers and solid metal containing nanoparticles.

\subsection{Phase contrast imaging of gold nanoparticles in lung tissue}

Schittny et al., [32] are currently using the phase contrast capabilities of TOMCAT to investigate gold nanoparticles (GNP) uptake in adults rats. They have combined classical absorption-based tomographic microscopy with modified transport of intensity approach [33] to obtain high resolution visualization of lung tissue down to the single alveoli.

Figure 5 shows a 3D representation of the GNP within the lung tissue of an adult rat. Animals have been sacrificed at different time laps after inhalation of GNP. The left panel shows a 3D rendering of the GNP-clusters obtained in absorption contrast mode. No lung tissue is visible because of its extremely weak absorption. The central panel shows the air-tissue interface, this time visible since phase contrast imaging has been used [33]. The right panel shows virtual, orthogonal slices thorough the alveolar wall: the GNP clusters can be clearly identified. It is interesting to note here that, albeit the animal has been sacrificed only 30 minutes after inhalation, GNP are found inside the alveoli walls.

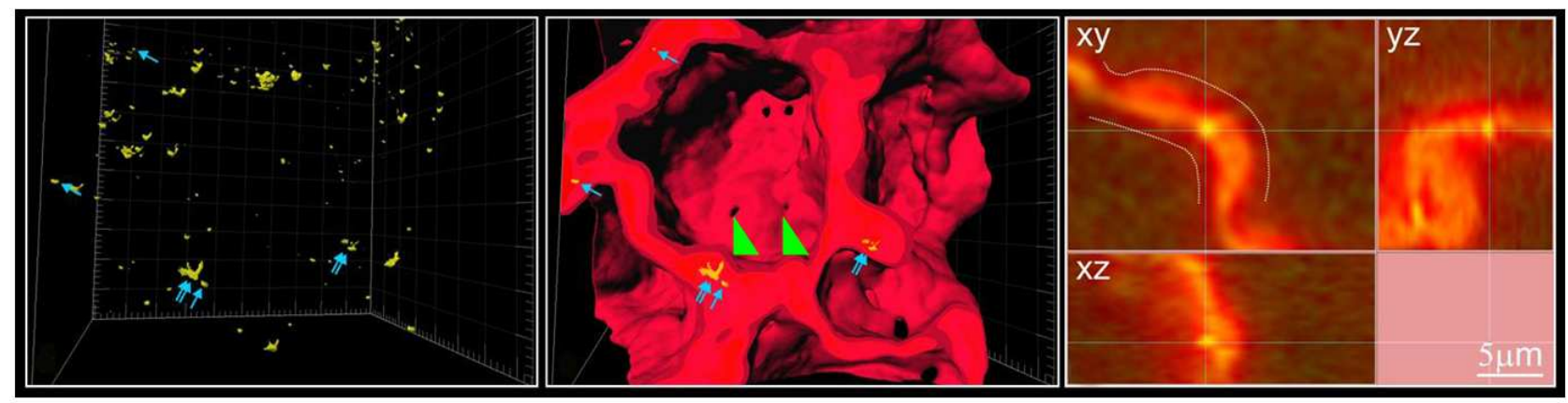

Figure 5: Left: Rendering of GNP clusters, in absorption contrast. The particles labeled with the arrows are also visible on the central panel, because all others are hidden inside the septa. Middle: Surface rendering of the air-tissue interface. After the exposure all the particles were observed inside the septa (arrows at cut surfaces of the septa). The green arrow heads show inter-alveolar Kohn's pores. Right: Orthoslices through one alveolar wall, clearly showing the GNP uptake.

\section{MATERIALS PROPERTIES OF GAS DIFFUSION LAYERS}

\subsection{Background and motivation}

Polymer electrolyte fuel cells (PEFCs) are complex electrochemical reactors where the transport of mass, heat, and charge is interlinked on a wide range of scales. The understanding of transport properties and limitations on all scales is therefore of prime importance for the optimization of the involved structures and materials. On the level of the single- 
cell porous structures, the so-called gas diffusion layers (GDLs) are employed for collecting current over the channels and to provide access for the gases under the flow field ribs. Mass, charge, and energy are transported in and through the GDL structures in all directions in the pressure-concentration, potential, and temperature fields, respectively. The GDL porous materials typically consist of carbon fibers with a diameter of 6-8 microns, which often are sintered with a carbonaceous binder. The resulting pore size distribution usually lies in the range of tens of micrometers. Due to the orientation of the fibers the pore and solid structures are highly anisotropic. The structural anisotropy is reflected in anisotropic diffusivities, permeabilities, and conductivities, which strongly affect and limit the mass transport through the void [34] and charge transport in the solid. This in turn has a considerable influence on the local current distribution [35] and the integral fuel cell performance. Therefore, GDL materials have to fulfill, beyond chemical and mechanical stability, two antagonistic demands: they need to be highly permeable and highly conductive. These transport properties are described by the effective material properties: conductivity, diffusivity, and permeability. The accurate knowledge of these parameters is required to improve the power density and efficiency of PEFCs. Becker et al. [4] recently described how simulations based on phase contrast X-ray tomographic microscopy data can be used to design GDLs with special properties which, in turn, are verified by real experiments. Essentially, based on the three-dimensional images of the sample, permeability, diffusivity, and conductivity are numerically determined by solving the partial differential equations describing flow, diffusion, and conduction, respectively and the results are compared with the measurement.

\subsection{Methods}

Diffusimetry, Permeametry and conductivity measurements

Binary diffusion of species $a$ and $b$ in a porous medium can be described by Fick's first law and effective media theory [36] stating that $j=-D_{a b}^{e f f} \nabla c$, where $c$ is the concentration, $j$ is the molar flux, and $D_{a b}^{e f f}$ is the effective diffusion coefficient tensor. The latest is split into a form factor $\varepsilon / \tau$ and a bulk diffusivity $D_{a b}$ according to $D_{a b}^{e f f}=\frac{\varepsilon}{\tau} D_{a b}$. $\varepsilon$ is the porosity and $\tau$ is the tortuosity, a measure for the connectivity of the pores. Diffusimetry has been performed according to Kramer et al. [37].

Convection in a porous medium can be approximated by Darcy's law. The velocity of the fluid $\mathbf{u}$ is proportional to the

gradient of total pressure $\nabla p$, according to $\mathbf{u}=-\frac{\kappa}{\mu} \nabla p$ where $\kappa$ is the permeability tensor of the porous media and $\mu$ is the dynamic viscosity of the fluid. The effect of in-plane compression on the permeability have been measured according to Chang et al. [38].

Electron transport in porous media can be described by Ohm's law combined with effective media theory. The current density $j$ is inversely proportional to the potential gradient $\nabla \Phi$ with $\sigma_{\text {eff }}$ being the effective electric conductivity so that $j=-\sigma_{\text {eff }} \nabla \Phi$.

\section{Structure Model}

A prerequisite of the numerical simulations is the construction of a 3D structure model. In Becker et al. [4], a structure model is a 3D rectangular area $\Omega$ subdivided in cubic voxels. Each voxel represents either solid material or pore space. The pore space is termed $\Omega_{P}$ and the solid is $\Omega_{S}$ so that $\Omega=\Omega_{P} \cup \Omega_{S}$. If such a model is given, the diffusivity, permeability, and conductivity are determined using homogenization theory [39]. It is assumed that the model shows a representative part of the GDL. Finally transport coefficient of the macroscopic equation (Darcy's law, Fick's law, and Ohm's law) is determined by solving the corresponding transport problem in the structure model. In the model described by Becker et al. [4], Toray TGP-H-060 gas diffusion material containing both fibers and binder has been used. In the virtual model, it was assumed that both make up about $50 \mathrm{wt} \%$. In a first step, fibers were added using Poisson line process until the desired porosity was reached. In a second step, the binder was added until $50 \mathrm{wt} \%$ was reached. Addition of the binder followed the idea that the binder basically behaves like a wetting fluid, therefore filling small pores first. Figure 6 shows a model created this way. Structural information has been obtained with phase contrast 
tomographic microscopy, using a modified transport of intensity approach [40]. Energy was $30 \mathrm{keV}$ and the microscope was operated with the 10x objective. The CCD camera was un-binned, covering a field of view of approx. $1.5 \times 1.5 \mathrm{~mm}$.
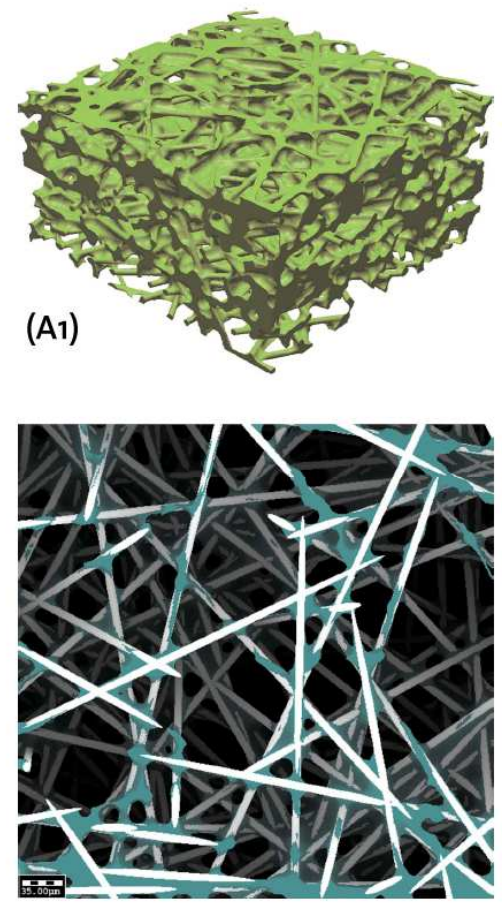

(A2)
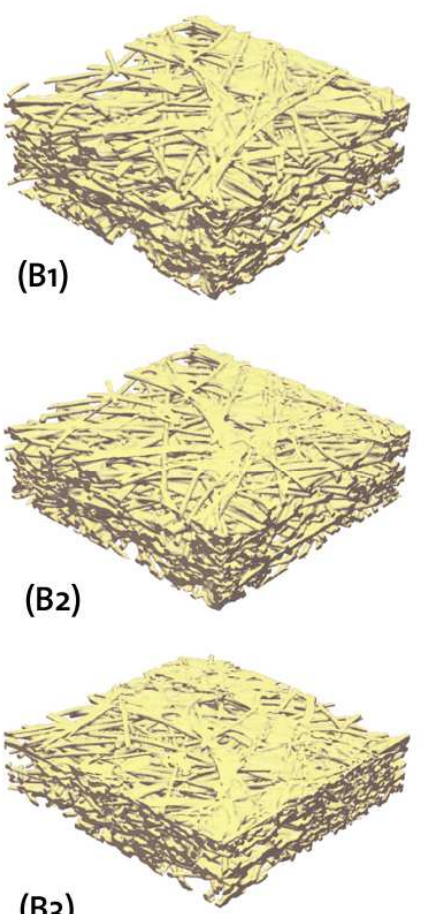

(B3)

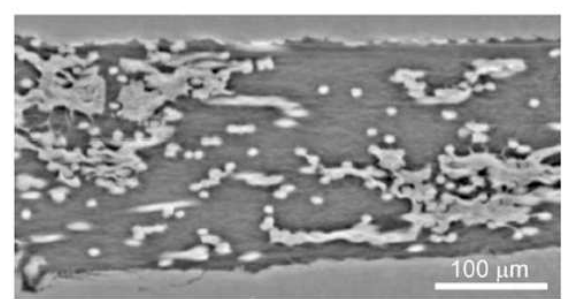

(C1)

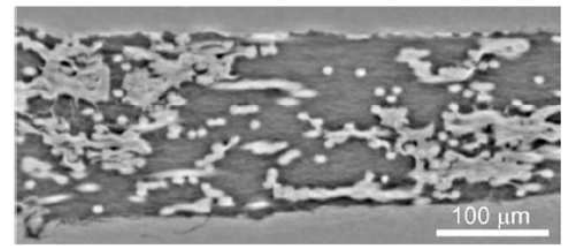

(C2)

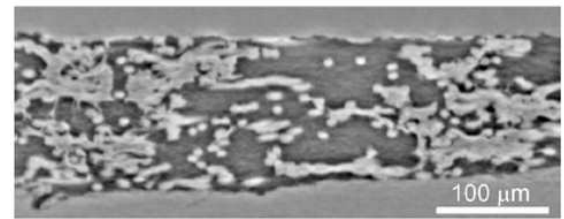

(C3)

Figure 6: (A1-A2): Virtually created GDL model showing an uncompressed carbon paper (A1). The model has a size of $600 \times 600 \times 300$ voxels, with 0.7 microns is size. (A2) Cross-section through the sample showing fiber (white) and binder (turquoise). (B1-B3): Reconstructed 3D structure of TGP-H-060 at three different compression levels based on the tomographic images shown in (C1-C3). The height varies with the rate of compression: (B1) 156.8, (B2) 119, and (B3) 98 microns, with porosities of 73.82, 69.49 and 66.78\% respectively. (C1-C3) Tomographic reconstruction of the GDL at different compression levels: (C1) 210, (C2) 190 and (C3) 150 microns. Figures adapted from [4] with permission.

\section{HARD X-RAYS PHASE CONTRAST IMAGING PERSPECTIVES}

Experimental data used for the showcases described in sections 2 and 3 have been obtained with established, state-of-theart tomographic microscopy techniques based on absorption. Sections 4 and 5 have been exploiting phase contrast imaging as a new tool. In the future, phase sensitive techniques will be massively used as a new, powerful investigation method: both high (density) sensitivity and high (spatial) resolution will be required. For this purpose, at the TOMCAT beamline of the Swiss Light Source, we are actively developing hard X-rays phase contrast capabilities [41] . This novel contrast regime is evolving rapidly and there is the need for cutting-edge instrumentation able to sense phase signals at different length scales. This last section briefly describes how TOMCAT has been equipped for this challenge and shows two examples illustrating high sensitive phase contrast imaging from the 10 microns down to 0.1 microns length scale.

\subsection{Grating interferometry}

For moderate spatial resolution requirements ( 5 to 10 microns) TOMCAT operates a two-gratings interferometer, as described in McDonald et al. [42]. In this instrument, a silicon phase grating divides the incident X-ray beam into the first two diffraction orders, which, through the Talbot effect, form a periodic interference pattern in the plane of the a 
second analyzer grating. A phase object placed in the incident beam will cause slight refraction and therefore modifications of the original wavefront profile. These variations result in changes of the locally transmitted intensity through the analyzer containing quantitative information on the phase gradient of the object. To separate the phase information from other contributions, either a phase-stepping approach [43] or a reverse-projection method is used [44].
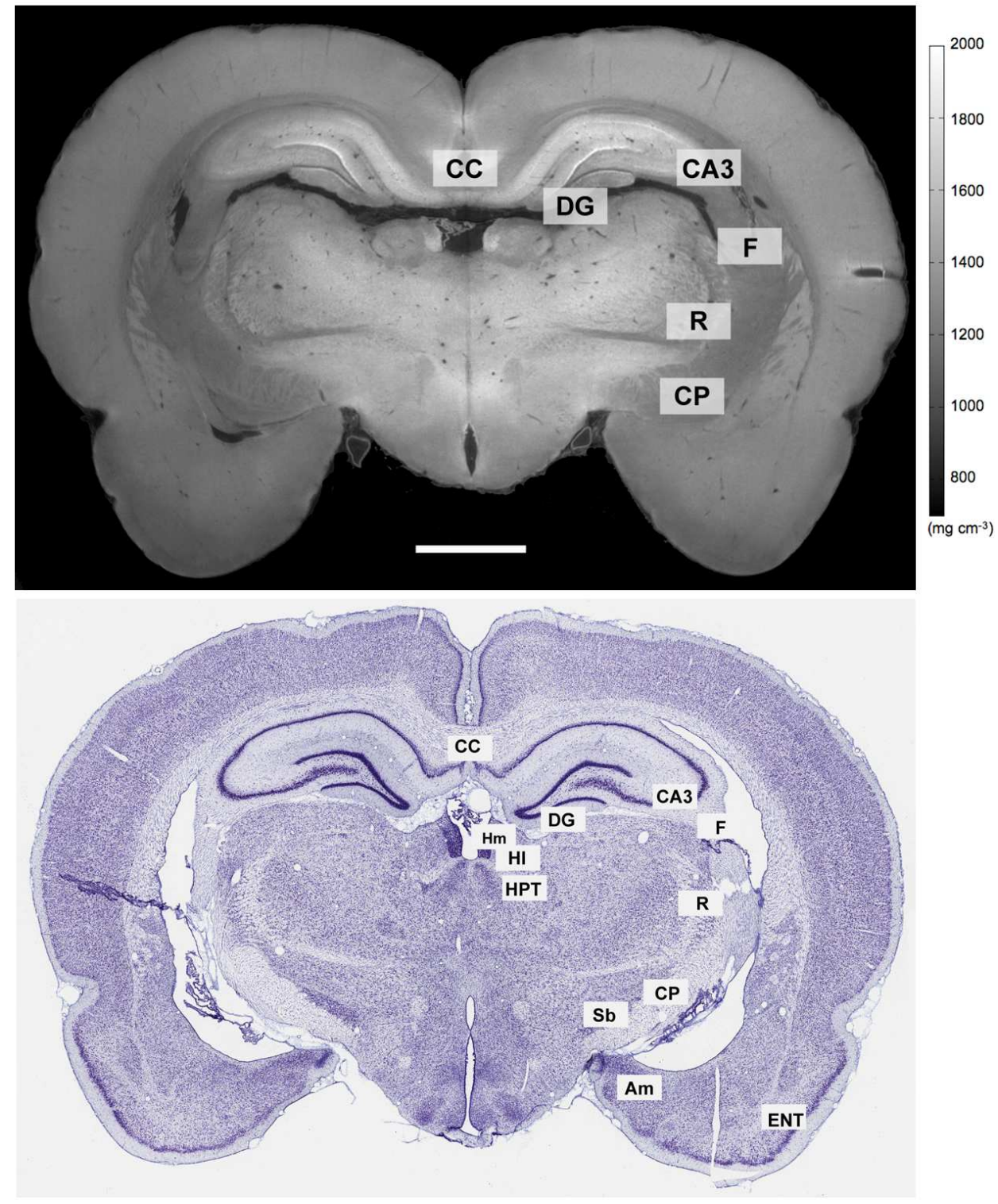

Figure 7: Top: Tomographic reconstruction (coronal) of a rat brain, fixed in paraffin, obtained with the TOMCAT interferometer operated at $25 \mathrm{keV}, 3^{\text {rd }}$ Talbot order. Spatial resolution is around 10 microns. Sensitivity: $1 \mathrm{mg} / \mathrm{cm}^{3}$. Scale bar: $1 \mathrm{~mm}$. Bottom: Nissl-stained histological section of the same region. Data from: www.brainmaps.org .

Figure 7 shows a coronal slice through a rat brain, at the height of the hippocampus, obtained with the TOMCAT interferometer. The brain was fixed in $4 \%$ paraformaldehyde and finally embedded in paraffin. With an isotropic spatial resolution of $\sim 10$ microns, several of the most relevant hallmarks are clearly visible: the corpus callosum (CC), cornu ammonis 3 (CA3), dentate gyrus (DG), formi of the hippocampus (F), reticular nucleus of the thalamus (R), and cerebral pedincule $(\mathrm{CP})$, to name just a few. 


\subsection{Zernike-phase contrast $\mathrm{X}$-ray nanoimaging}

Spatial resolution between 0.1 and 0.5 microns are reached by implementing a hard X-ray full-field microscope. For this instrument, the key optical elements are a condenser, to provide illumination of the sample, and an objective lens, to produce a magnified image of the sample on the detector. The instrument has been designed to provide homogeneous and intense illumination numerically matched with the objective lens [45]. We obtained phase contrast by adapting Zernike's traditional principle of using hollow cone illumination and an imaging objective in combination with a separate $\pm \pi / 2$ phase ring to our particular illumination geometry. In our case at the back focal length of the lens the phase ring had to be replaced by a phase dots array [6]. A 3D resolution of $144 \mathrm{~nm}$ has been measured.

Figure 8 displays X-ray nanoradiography (a) and nanotomography (b-c) images of MC3 preosteoblast cells-fixed but not stained-contained in a glass capillary (100 microns diameter, 10 microns wall thickness) and suspended in a $1.3 \mathrm{wt} \%$ hydrogel. The measurement has been done at $10 \mathrm{keV}$.

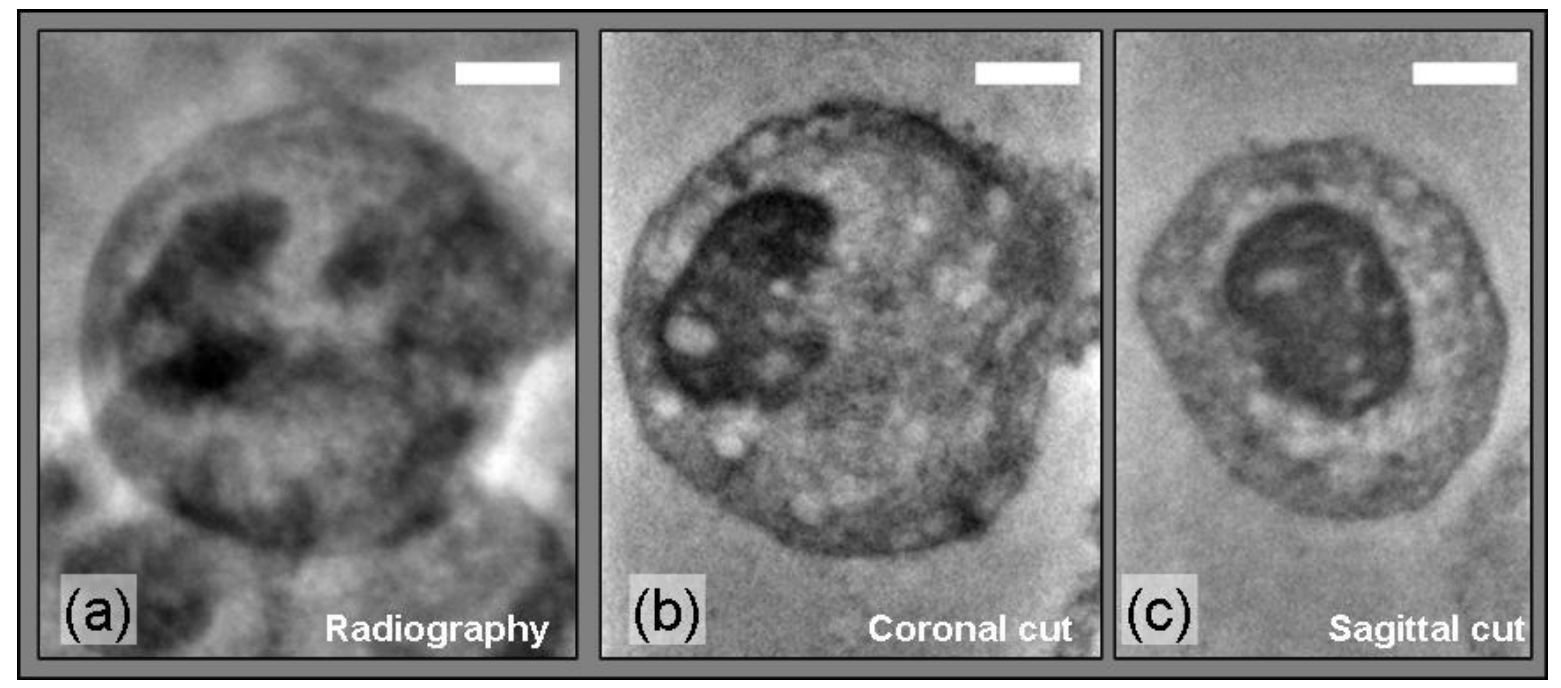

Figure 8: Zernike phase contrast nanotomography of an unstained MC3 preosteoblast cell. (a) shows a nanoradiographic projection of the MC3 cell together with other cells which happened to be on the beam path during the snapshot. (b) and (c) show coronal and sagittal cut of the MC3 cell, respectively. Clearly visible are the nucleus (dark) with several intracellular structures. Scale bar are 5 microns. (c) Americal Physical Society. 


\section{REFERENCES}

[1] M. Stampanoni, A. Groso, A. Isenegger et al., "TOMCAT: A beamline for TOmographic Microscopy and Coherent rAdiology experimenTs," Synchrotron Radiation Instrumentation, Pts 1 and 2, 879, 848-851 (2007).

[2] J. Reichold, M. Stampanoni, A. L. Keller et al., "Vascular graph model to simulate the cerebral blood flow in realistic vascular networks," Journal of Cerebral Blood Flow and Metabolism, 29(8), 1429-1443 (2009).

[3] A. Tsuda, N. Filipovic, D. Haberthur et al., "Finite element 3D reconstruction of the pulmonary acinus imaged by synchrotron X-ray tomography," Journal of Applied Physiology, 105(3), 964-976 (2008).

[4] J. Becker, R. Fluckiger, M. Reum et al., "Determination of Material Properties of Gas Diffusion Layers: Experiments and Simulations Using Phase Contrast Tomographic Microscopy," Journal of the Electrochemical Society, 156(10), B1175-B1181 (2009).

[5] W. H. De Jong, and P. J. Borm, "Drug delivery and nanoparticles:applications and hazards," Int J Nanomedicine, 3(2), 133-49 (2008).

[6] M. Stampanoni, R. Mokso, F. Marone et al., "Phase-contrast tomography at the nanoscale using hard x rays," Physical Review B, 81(14), 140105 (2010).

[7] M. F. Stampanoni M., Modregger P., Pinzer B., Thüring T. , Vila-Comamala J., David C., Mokso R., "Tomographic Hard X-ray Phase Contrast Micro- and Nano-imaging at TOMCAT," AIP Conference Proceedings, (2010).

[8] S. Ogawa, T. M. Lee, A. R. Kay et al., "Brain magnetic resonance imaging with contrast dependent on blood oxygenation," Proc Natl Acad Sci U S A, 87(24), 9868-72 (1990).

[9] K. K. Kwong, J. W. Belliveau, D. A. Chesler et al., "Dynamic magnetic resonance imaging of human brain activity during primary sensory stimulation," Proc Natl Acad Sci U S A, 89(12), 5675-9 (1992).

[10] R. Turner, "How much cortex can a vein drain? Downstream dilution of activation-related cerebral blood oxygenation changes," Neuroimage, 16(4), 1062-7 (2002).

[11] N. M. Tsoukias, D. Goldman, A. Vadapalli et al., "A computational model of oxygen delivery by hemoglobinbased oxygen carriers in three-dimensional microvascular networks," J Theor Biol, 248(4), 657-74 (2007).

[12] M. A. Castro, C. M. Putman, and J. R. Cebral, "Patient-specific computational modeling of cerebral aneurysms with multiple avenues of flow from 3D rotational angiography images," Acad Radiol, 13(7), 811-21 (2006).

[13] D. A. Boas, S. R. Jones, A. Devor et al., "A vascular anatomical network model of the spatio-temporal response to brain activation," Neuroimage, 40(3), 1116-29 (2008).

[14] F. Plouraboue, P. Cloetens, C. Fonta et al., "X-ray high-resolution vascular network imaging," J Microsc, 215(Pt 2), 139-48 (2004).

[15] C. B. Schaffer, B. Friedman, N. Nishimura et al., "Two-photon imaging of cortical surface microvessels reveals a robust redistribution in blood flow after vascular occlusion," PLoS Biol, 4(2), e22 (2006).

[16] P. Gehr, M. Bachofen, and E. R. Weibel, "The normal human lung: ultrastructure and morphometric estimation of diffusion capacity," Respir Physiol, 32(2), 121-40 (1978).

[17] J. C. Schittny, S. I. Mund, and M. Stampanoni, "Evidence and structural mechanism for late lung alveolarization,” Am J Physiol Lung Cell Mol Physiol, 294(2), L246-54 (2008).

[18] M. J. Cookson, C. J. Davies, A. Entwistle et al., "The microanatomy of the alveolar duct of the human lung imaged by confocal microscopy and visualised with computer-based 3D reconstruction," Comput Med Imaging Graph, 17(3), 201-10 (1993).

[19] D. Haberthur, C. Hintermuller, F. Marone et al., "Radiation dose optimized lateral expansion of the field of view in synchrotron radiation X-ray tomographic microscopy," Journal of Synchrotron Radiation, 17(5), (2010).

[20] C. Hintermuller, F. Marone, A. Isenegger et al., "Image processing pipeline for synchrotron-radiation-based tomographic microscopy," Journal of Synchrotron Radiation, 17(4), 550-559 (2010).

[21] J. C. Schittny, M. Paulsson, C. Vallan et al., "Protein cross-linking mediated by tissue transglutaminase correlates with the maturation of extracellular matrices during lung development," Am J Respir Cell Mol Biol, 17(3), 334-43 (1997).

[22] J. C. Schittny, V. Djonov, A. Fine et al., "Programmed cell death contributes to postnatal lung development," Am J Respir Cell Mol Biol, 18(6), 786-93 (1998). 
[23] D. Haberthür, [High resolution tomographic imaging of the alveolar region of the mammalian lung] University of Bern, (2010).

[24] R. Ardila, T. Horie, and J. Hildebrandt, "Macroscopic isotropy of lung expansion," Respir Physiol, 20(2), 10515 (1974).

[25] J. Sznitman, T. Heimsch, J. H. Wildhaber et al., "Respiratory Flow Phenomena and Gravitational Deposition in a Three-Dimensional Space-Filling Model of the Pulmonary Acinar Tree," Journal of Biomechanical Engineering-Transactions of the Asme, 131(3), (2009).

[26] H. Miki, J. P. Butler, R. A. Rogers et al., "Geometric hysteresis in pulmonary surface-to-volume ratio during tidal breathing," Journal of Applied Physiology, 75(4), 1630-6 (1993).

[27] A. Tsuda, F. S. Henry, and J. P. Butler, "Chaotic mixing of alveolated duct flow in rhythmically expanding pulmonary acinus," Journal of Applied Physiology, 79(3), 1055-63 (1995).

[28] S. Haber, and A. Tsuda, "The effect of flow generated by a rhythmically expanding pulmonary acinus on aerosol dynamics," Journal of Aerosol Science, 29(3), 309-322 (1998).

[29] J. Sznitman, R. Sutter, D. Altorfer et al., "Visualization of respiratory flows from 3D reconstructed alveolar airspaces using X-ray tomographic microscopy," Journal of Visualization.

[30] C. W. Lam, J. T. James, R. McCluskey et al., "Pulmonary toxicity of single-wall carbon nanotubes in mice 7 and 90 days after intratracheal instillation," Toxicol Sci, 77(1), 126-34 (2004).

[31] K. Donaldson, V. Stone, C. L. Tran et al., "Nanotoxicology," Occup Environ Med, 61(9), 727-8 (2004).

[32] J. C. Schittny, [In preparation], (2010).

[33] A. Groso, R. Abela, and M. Stampanoni, "Implementation of a fast method for high resolution phase contrast tomography," Optics Express, 14(18), 8103-10 (2006).

[34] R. Flückiger, S. A. Freunberger, D. Kramer et al., "Anisotropic, effective diffusivity of porous gas diffusion layer materials for PEFC," Electrochimica Acta, 54(2), 551-559 (2008).

[35] S. A. Freunberger, M. Reum, J. Evertz et al., "Measuring the Current Distribution in PEFCs with SubMillimeter Resolution," Journal of the Electrochemical Society, 153(11), A2158-A2165 (2006).

[36] Bruggeman, Ann. Phys., 24, 636 (1936).

[37] D. Kramer, S. A. Freunberger, R. Flückiger et al., "Electrochemical diffusimetry of fuel cell gas diffusion layers," Journal of Electroanalytical Chemistry, 612(1), 63-77 (2008).

[38] W. R. Chang, J. J. Hwang, F. B. Weng et al., "Effect of clamping pressure on the performance of a PEM fuel cell," Journal of Power Sources, 166(1), 149-154 (2007).

[39] Hornung, [Homogenization and Porous Materials], New York(1997).

[40] A. Groso, R. Abela, and M. Stampanoni, "Implementation of a fast method for high resolution phase contrast tomography," Optics Express, 14(18), 8103-8110 (2006).

[41] T. T. Stampanoni M.; Marone F; Modregger P.; Pinzer B., Vila-Comamala J., David C., Mokso R., "Tomographic Hard X-ray Phase Contrast Micro- and Nano-imaging at TOMCAT," AIP Conference Proceedings, (2010).

[42] S. A. McDonald, F. Marone, C. Hintermuller et al., "Advanced phase-contrast imaging using a grating interferometer," J Synchrotron Radiat, 16(Pt 4), 562-72 (2009).

[43] T. Weitkamp, A. Diaz, C. David et al., "X-ray phase imaging with a grating interferometer," Optics Express, 13(16), 6296-304 (2005).

[44] Z. K. Zhu P., Wang Z., Liu Y., Liu X., Wu Z., McDonald S. A., Marone F., Stampanoni M., "Low dose, single step, grating based X-ray phase contrast imaging,,” Proc Natl Acad Sci U S A, (2010).

[45] K. Jefimovs, J. Vila-Comamala, M. Stampanoni et al., "Beam-shaping condenser lenses for full-field transmission X-ray microscopy,” J Synchrotron Radiat, 15(Pt 1), 106-8 (2008). 